\title{
Gene regulation network analyses of pistil development in papaya
}

\author{
Zhenyang Liao ${ }^{1}$, Fei Dong ${ }^{1}$, Juan Liư², Lele Xu' ${ }^{1}$ Amy Marshall-Colon ${ }^{3}$ and Ray Ming ${ }^{3 *}$
}

\begin{abstract}
Background: The pistil is an essential part of flowers that functions in the differentiation of the sexes and reproduction in plants. The stigma on the pistil can accept pollen to allow fertilization and seed development. Papaya (Carica papaya $\mathrm{L}$.) is a dioecious plant, where female flowers exhibit normal pistil, while the male flowers exhibit aborted pistil at a late stage of pistil development.

Results: The developmental stages of papaya pistil were analyzed after first dividing it into slices representing the primordium stage 1 (S1), the pre-meiotic stages $\mathrm{S} 2$, post-meiotic stage $\mathrm{S} 3$, and the mitotic stage $\mathrm{S} 4$. The SS scoring algorithm analysis of genes preferentially expressed at different stages revealed differentially expressed genes between male and female flowers. A transcription factor regulatory network for each stage based on the genes that are differentially expressed between male and female flowers was constructed. Some transcription factors related to pistil development were revealed based on the analysis of regulatory networks such as CPAGL11, CPHEC2, and CpSUPL. Based on the specific expression of genes, constructed a gene regulatory subnetwork with CPAGL11-CpSUPL-CpHEC2 functioning as the core. Analysis of the functionally enriched terms in this network reveals several differentially expressed genes related to auxin/ brassinosteroid signal transduction in the plant hormone signal transduction pathway. At the same time, significant differences in the expression of auxin and brassinosteroid synthesis-related genes between male and female flowers at different developmental stages were detected.
\end{abstract}

Conclusions: The pistil abortion of papaya might be caused by the lack of expression or decreased expression of some transcription factors and hormone-related genes, affecting hormone signal transduction or hormone biosynthesis. Analysis of aborted and normally developing pistil in papaya provided new insights into the molecular mechanism of pistil development and sex differentiation in dioecious papaya.

Keywords: Carica papaya, Pistil development, Gene regulatory network, Transcription factor

\section{Background}

Flowers are the reproductive organs of flowering plants, and their development involves complex regulatory processes. The precise control of the expression of flower development-related genes guides the formation of four whirls so that angiosperms exhibit a wide variety of flower morphologies [1]. The carpel is a unique

\footnotetext{
${ }^{*}$ Correspondence: rayming@illinois.edu

${ }^{3}$ Department of Plant Biology, University of Illinois at Urbana-Champaign, Urbana, IL 61801, USA

Full list of author information is available at the end of the article
}

reproductive structure in angiosperms that contains ovules and female gametophytes. The stigma at the top of the carpel facilitates pollen recognition and pollen tube elongation, and the carpel not only protects ovules from insects or microorganisms but also nurtures the development of plant male and female gametophytes. After fertilization, carpels develop into fruit.

Each step of flower organ development is genetically regulated, and a network of finely regulated genes directs the elaboration of flower morphology through temporal and spatial expression and interactions among specific genes [2]. Among these, the AGAMOUS 
(AG) gene plays a crucial role in the regulatory network of flower development [2]. AG is a C-class functional gene in the ABCDE model of flower development $[3,4]$ and plays an important role in regulating the development of stamens and carpel primordia [5]. SEEDSTICK (STK), also known as AGAMOUS-LIKE 11 (AGL11), is a D-class functional gene and transcription factor MADS-box family in the ABCDE model [6], which are essential for ovule development [7]. Silencing the homologs of $A G$ and $S T K$ in poplars leads to changes in the characteristics of floral organs and affects the determination of floral organs, ovule differentiation, and seed hair development [8]. Further, AGL11 also plays an essential regulatory role in ovule development and seed formation in tomato, grape, rice, and other species [9, 10]. SUPERMAN (SUP) is a regulator of flower homeotic genes and a transcription factor of the $\mathrm{C} 2 \mathrm{H} 2$-type zinc finger motif [11]. The SUP gene in Arabidopsis thaliana determines the boundary between the thirdand fourth-round organs and the growth of the integument, which is the cell layer surrounding the ovule [12]. SISUP is a SUP-like gene that is expressed exclusively in female flowers and involved in ovule development in white campion Silene latifolia Poiret [13]. The function of MdSUP11, one of the 12 SUPL genes in apple (Malus domestica Borkh.), is related to vegetative and reproductive development in apples. Its overexpression affects the development of leaf and floral organs and plant height in tobacco [14].

The development of carpels and fruit are also directly or indirectly regulated by plant hormones. For example, in maize, a low concentration of jasmonic acid (JA) can promote the development of female organs and inhibit the development of male organs, resulting in abnormal development of seeds in tassels, which can be reversed by the application of JA $[15,16]$. Ethylene can promote the development of cucumber carpels. In hermaphrodite cucumbers, a gene encoding ACC oxidase participates in carpel development by regulating the ethylene content, and ethylene-induced ACS11 is also crucial for carpel development [17]. The numbers of ovules and seeds in Arabidopsis are positively regulated by brassinosteroid signaling [18]. Brassinosteroid controls the number of ovules by influencing the expression of the gene BZR1 on ovule development. The $b z r 1-1 D$ mutant (the gainof-function of BZR1) increased the number of ovules and seeds, while the brassinosteroid-deficient mutant det2-1 produced fewer ovules and seeds [19]. At the same time, brassinosteroids can regulate the number of ovules by down-regulating gibberellins biosynthesis, thereby promoting the stability of DELLA protein, which will eventually promote the initiation of ovule primordia in tomatoes [20].
Auxin is another critical plant hormone regulator of carpel development in flowers. In Capsella bursa-pastoris L., the polar distribution of auxin at the top of the carpel maintains a high concentration of auxin in the stigma, and this differential distribution of auxin promotes morphogenesis of the carpel [21]. Lateral transport of auxin can promote increased cell division and thereby cause the carpel to bend [22]. Also, decreased capacity for longitudinal polar auxin transport in the carpel can directly affect carpel length [23]. Further, auxin is distributed in a concentration gradient at different locations in developing plant structures. Thus, the observed differences in carpel morphology between C. bursa-pastoris and Arabidopsis are due to differences in the polar distribution of auxin during carpel development [21].

Dioecious plant species have been derived from hermaphroditic ancestral species in which a mechanism of stamen or pistil abortion evolved to prevent selfpollination. The resulting plant species with gynodioecious or androdioecious individuals eventually evolved into species with dioecious individuals [24]. During this evolutionary process, genes that regulate pistil or stamen abortion became the key genes for plant sex determination. Papaya (Carica papaya L.) is a rare trioecious plant species that can bear female, male, or hermaphrodite flowers on separate plants. Sex determination in papaya is controlled by pairs of sex chromosomes, for which $\mathrm{XX}$ represents female, $\mathrm{XY}$ represents male, and $\mathrm{XY}^{\mathrm{h}}$ represents hermaphrodite plants [25] that originated about 7 million years ago. An $8.1 \mathrm{Mb}$ sex-determination region has been detected in the $\mathrm{Y}^{\mathrm{h}}$ chromosome (HSY), while the corresponding region in the $\mathrm{X}$ chromosome is $3.5 \mathrm{Mb}$ in length [26]. Homologous sequence comparison between HSY and X reveals two evolutionary strata, corresponding to two chromosomal inversions on HSY that occurred 7 and 1.9 million years ago, respectively. The gene annotation of HSY revealed that there are 72 protein-coding genes, 50 of which have homologous alleles in the $\mathrm{X}$ corresponding homologous region, and the other 22 are HSY-specific genes. There are 84 genes in the $X$ region with $34 \mathrm{X}$-specific genes [26, 27]. Similarly, a sex-determination region has also been detected on the Y chromosome (MSY) in male papaya, and the MSY and HSY regions share $99.6 \%$ similarity [28]. Papaya sex-determination genes are located within this region, as would be expected according to the theory of sex chromosome evolution in plants [29]. The female flowers of papaya have well-developed pistils and can accept pollen to form fruits. In contrast, male flowers cannot form fruits due to aborted pistil.

The pistils of papaya male and female flowers exhibit obviously distinct morphology, which provides an excellent opportunity to study the genetic mechanisms that 
control pistil development and sex determination in papaya. Here, RNA-Seq technology was used to analyze the pistil transcriptomes of female and male papaya flowers at different developmental stages. These data were used to determine the transcriptional differences between pistils in flowers of different sexes and to explore transcriptional regulation related to pistil development. We constructed a gene regulation subnetwork for carpel development in papaya based on the specificity of gene expression. Finally, candidate genes were identified for their possible important roles of auxin and brassinosteroid biosynthesis and signal transduction in the molecular mechanisms of sex differentiation and carpel development in papaya.

\section{Results}

\section{Global transcriptome analysis of pistil development in female and male papaya flowers}

Male papaya flowers bear an aborted pistil, while the pistils of the female flowers develop normally. Global transcription analysis of different developmental stages of female and male flower carpels can reveal the molecular mechanism of male flower carpel abortion. The development of papaya flowers was divided into four stages (Fig. 1). These four stages of pistil development represent major events, including flower primordium formation, carpel formation, meiosis, and mitosis. Stage 1 is the representative stage for flower primordium formation, the key stage for papaya sex determination when both male and female flowers are smaller than $1 \mathrm{~mm}$ in length. There is no difference in the morphology of male and female flowers in this stage (Fig. 1). Stage 2 and 3 flowers are $1-2.5 \mathrm{~mm}$ and $2.5-4.5 \mathrm{~mm}$ in length, respectively.
At these stages, the male and female flowers form carpels with important morphological differences and continue to develop. Multiple normal carpels are formed in female flowers, while only one pointed carpel is formed in male flowers (Fig. 1). The Stages 2 and 3 of carpel development coincide with meiosis in papaya ovules. During stage 4, ovules and ovaries the $4.5-6 \mathrm{~mm}$ flowers enter the mitotic stage, and the carpels of the female flowers coalesce to form the normal pistil, while the male flowers abort pistil. A normal pistil is formed with an enlarged base during this stage in female flowers, of which stigma, style, and ovary are all developing normally, but a particularly slender aborted pistil is formed in male flowers (Fig. 1).

To detect candidate genes that might be involved in sex determination and carpel abortion in male papaya flowers, transcriptomes were analyzed from female and male flower primordia and carpels at these different developmental stages. The high-quality reads number in different pistil development stages ranged from 27 to 58 million in female flowers, and 34 to 90 million in male flowers. We also obtained 180/197 and 38/302 million high-quality reads on female and male leaves, respectively (Additional file 16). In all, more than 1.1 billion high-quality paired-end reads were obtained from different tissues, with an average of about 40 million reads per sample. The high-quality reads were mapped to the papaya reference genome. The range of mapped reads is 27 to 294 million, accounting for 93.13 to $98.01 \%$ of the total reads (Additional file 16). We used the Spearman correlation coefficient to estimate the consistency and repeatability of the sequencing samples. The closer the value is to 1 , the stronger the correlation among replications. The Spearman correlation coefficient between expression values in

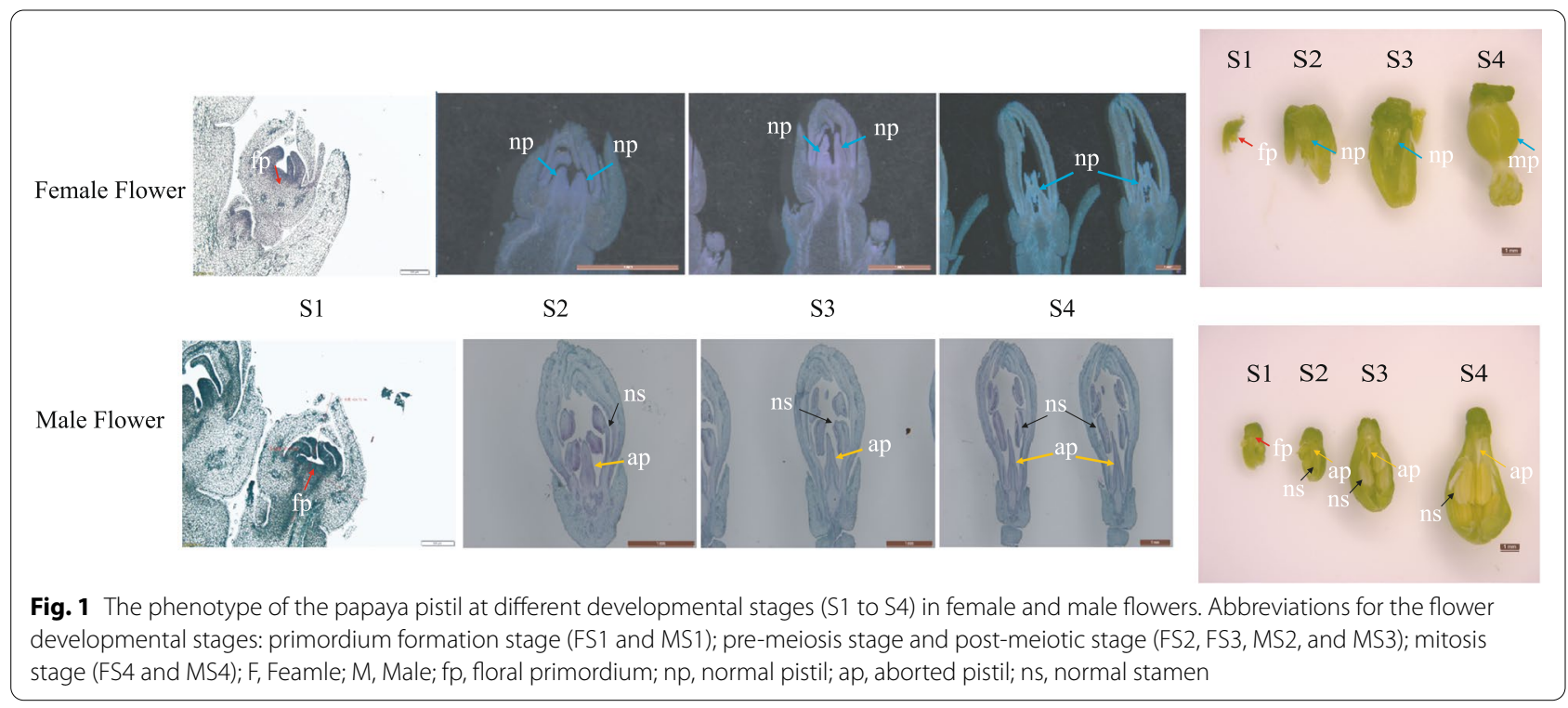


different biological replicate samples varied from 0.94 to 0.98 in female flowers and from 0.92 to 0.99 in male flowers, indicating high-quality data replication (Additional file 1).

In total, about $91 \%$ of sequenced papaya genes were expressed in at least one of the ten samples. The percentage of genes expressed in different pistil development stages ranged from $80 \%$ (FS2) to $82 \%$ (FS1) in female flowers, and $82 \%$ (MS1) to $86 \%$ (MS3) in male flowers. From 4.18 to $5.37 \%$ of the genes showed extremely high (FPKM $\geq 100$ ) transcript expression in the various tissues analyzed (Additional file 2a). The number of genes with high $(50 \leq \mathrm{FPKM}<100)$, medium $(10 \leq \mathrm{FPKM}<50)$, or low $(1 \leq$ FPKM $<10)$ transcript expression was similar in all pistil tissues (Additional file $2 \mathrm{~b}$ ). These analyses reflect effective transcriptome coverage of genes expressed during pistil development, which was beneficial for downstream analysis.

\section{Transcriptional analysis reveals relationships} between different stages of pistil development in papaya

To investigate global transcriptome differences during pistil development in female and male papaya flowers, PCA and SCC cluster analyses were performed based on the average FPKM value of all expressed genes in 10 samples. Higher correlations detected in these analyses might reflect more consistent transcriptional activity in various tissues or developmental stages. As expected, these analyses showed that the leaf transcriptomes of the male and female plants clustered together and differed significantly from the other tissues (Fig. 2). Significant differences in transcript abundances between different sexes at the early stage (FS1 and MS1) imply differences in transcriptional regulation activities in stage 1 between male and female flowers (Fig. 2b). The middle stages of pistil development (F/MS2 and F/MS3) showed very close clustering within sexes, indicating that their transcriptional programs were very similar at those times (Fig. 2b). Because meiosis is underway during both of these stages, combined them into one stage for downstream analysis. Interestingly, the transcriptional activity of the middle stage (F/MS2 and F/ MS3) clustered more closely with that of the early stage (MS1) in male flowers, but more closely with that of the late-stage (FS4) in female flowers. In the later stage of pistil development (F/MS4), large differences in transcriptional activities were observed in male and female flowers (Fig. 2b). The large differences in transcription programs at F/MS4 might ultimately determine the morphology and fertility of pistils. Taken together, these results indicate that male and female papaya flowers exhibit greater differences in transcription programs at particular stages of pistil development, which helps to direct further exploration of the growth and development of pistils at the transcriptional level.

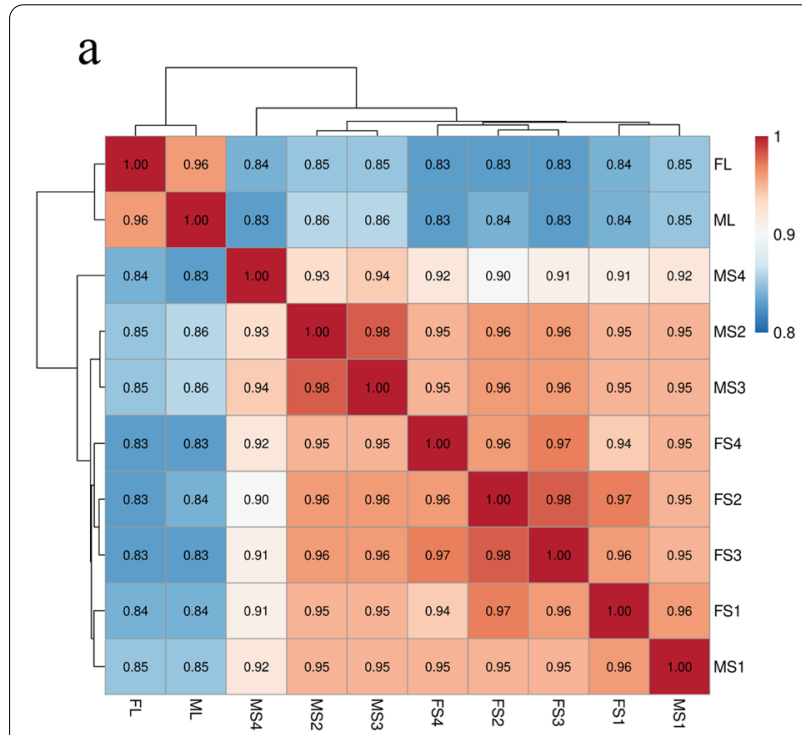

b

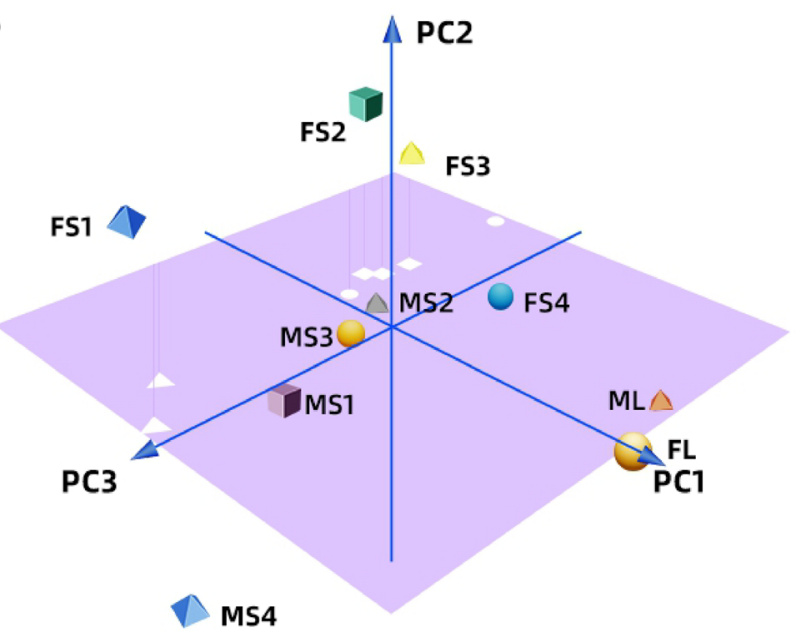

Fig. 2 The transcriptional correlation of pistil at different developmental stages in female and male flowers. a SCC was performed to analyze transcripts expressed in leaves and at four developmental stages of the pistil in female and male papaya flowers, and the redder the color, the stronger the correlation. The legend on the right represents the correlation coefficient. $\mathbf{b}$ PCA cluster analysis of leaves and pistils in different stages, different development stages are represented by 3D shapes in different colors 


\section{Preferentially expressed genes during pistil development in papaya}

To investigate transcriptional activities during different stages of carpel development in male and female flowers, genes that are preferentially expressed at particular stages of carpel development were defined. A 'stage-specific' (SS) algorithm was used to define the genes preferentially expressed by each gender at a specific developmental stage. According to this criterion, the number of preferentially expressed genes were detected ranged from 125 to 911 in female flowers and from 223 to 982 in male flowers depending on the stage (Fig. 3a and Additional file 3). The differences in the profiles of preferentially expressed genes at each developmental stage suggest that different stages or samples exhibit independent growth and developmental processes.

Gene ontology (GO) term analysis of all the preferentially expressed genes revealed many common processes and few unique processes across stages of development (Fig. 3b). Unique processes include cellular organism development and the development of anatomical structures in male or female flowers during early development (F/MS1). The unique processes were found during latestage development (FS4), including cell wall organization or biogenesis in the female flowers, macromolecule localization, glycosylation, cellular localization and cellular homeostasis in male flowers, and cellular component organization and cell communication in flowers of either sex. Genes expressed during the mid-stage of development were not enriched in unique GO terms.

Genes encoding 230 and 236 transcriptional regulators were uncovered in female and male flowers, respectively, of which 132 transcription factors (TFs) are shared by both transcriptomes (Additional file 4). The number of genes encoding TFs from one TF family (e.g., ERF, MADS, and HD-ZIP) expressed at different developmental stages varied between male or female flowers. Within a developmental stage, the number of expressed genes encoding TFs from one TF family differed between female and male flowers (Additional file 4). For example, the number of genes encoding MADS TFs expressed in female flowers was greater than that expressed in male flowers in the early stage (MS1), while genes encoding MYB TFs showed the opposite expression pattern. The fewer TFs were preferentially expressed in the middle stages (F/MS2 and F/MS3) of carpel development were detected, implying that the transcriptional program for TF-encoding genes is not very active at that time.

To verify the accuracy of our stage-specific (SS) expression algorithm, we randomly chose 24 preferentially

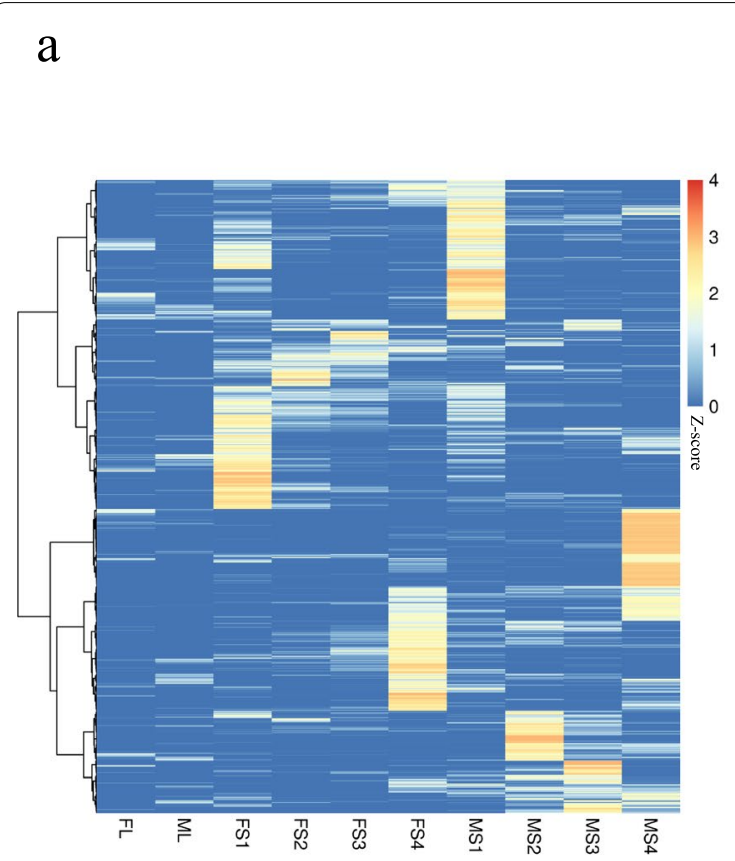

b

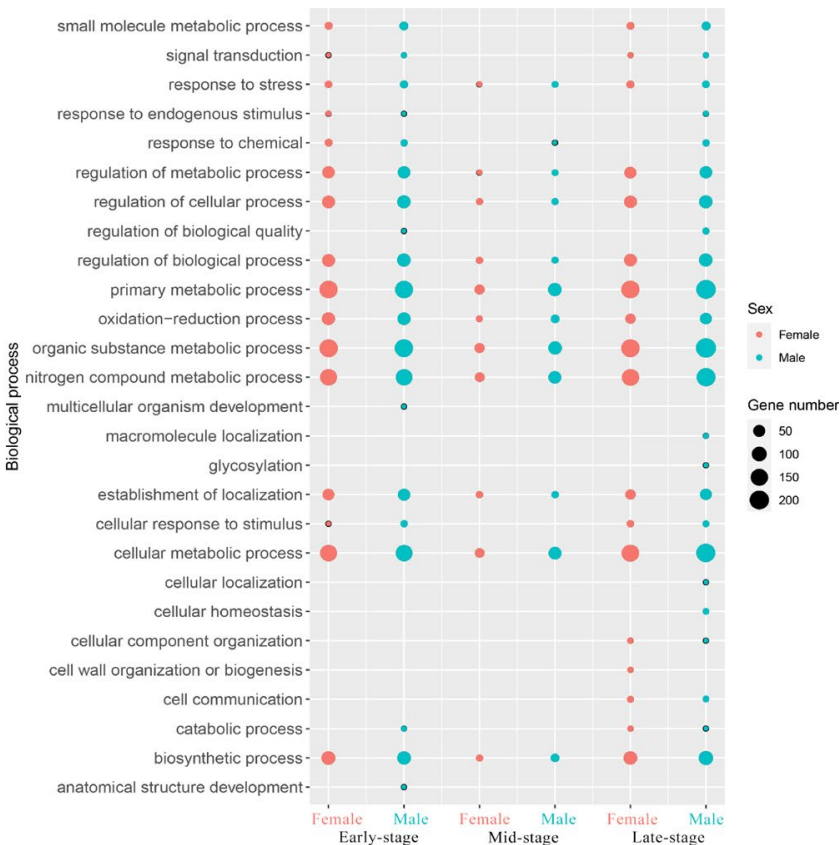

Fig. 3 Preferential gene expression between male and female papaya flowers during different stages of pistil development. a, Heatmap of preferential gene expression at different stages of pistil development in female and male papaya flowers. The larger the Z-score value, the redder the color and the higher the gene expression $\mathbf{b}$, The putative functions of genes preferentially expressed at different stages investigated by Gene Ontology (GO) term analysis. Different colors represent different sex types, and the size of the circle represents the number of genes 
expressed genes and performed quantitative reverse transcription PCR (RT-qPCR) experiments to analyze their expression at different developmental stages in females and males flowers. RT-qPCR verification of the expression of these genes showed that 19 (79.2\%) of the SS genes could be verified as consistent with the results of our RNA-Seq analysis (Additional file 5).

\section{Analysis of differentially expressed genes and construction of a gene regulatory network for papaya flower pistil development}

To study the transcript differences characteristic of different stages of female and male flower development in papaya, differentially expressed genes (DEGs) in each stage were identified using DESeq [30] and edgeR [31] (Additional file 6). In total, we identified 7165 and 8074 DEGs using DESeq and edgeR, respectively. A total of 7058 DEGs were shared between both male and female tissues, including 1397 in FS1_VS_MS1; 1403 in FS2_VS_ MS2; 1386 in FS3_VS_MS3; and 2872 in FS4_VS_MS4. These shared DEGs were then subjected to gene regulatory network (GRN) analysis. The analysis of the gene regulatory networks during pistil development in different sex type flowers provided a clearer understanding of the occurrence and regulation of the pistil development process. Based on the results of PCA analysis and RNASeq of tissue sections, S2 and S3 as the meiosis stage were combined together for further analysis.

The distribution of differentially expressed TF (DETF) families at particular stages was characterized (Additional file 7). During the FS1, FS2-3, and FS4 stages, 73, 104, and 140 up-regulated DETFs were identified, respectively, while during the MS1, MS2-3, and MS4 stages, 29, 112 , and 116 up-regulated DETFs were found, respectively. Notably, the top three gene families encoding DETFs were the bHLH, MYB, and MADS-box families. Interestingly, some of these TF-encoding gene families were expressed only in male or female flowers. Transcripts of genes encoding members of the ARR-B, B3, C3H, E2F/DP, ERF, GATA, GRF, HB-other, LFY, LSD, NF-YA, SAP, and Whirly TF families were expressed in female flowers but were not in male flowers, while genes encoding members of the CAMTA, NF-YC, and Nin-like TF families expressed in male flowers but not in female flowers.

To study the GRNs that operate during papaya pistil development, co-expression networks for both female and male flowers at three different developmental stages were constructed using Pearson correlation coefficient (PCC) and mutual rank (MR) analyses. Only DEGs with PCC and MR values above 0.95 were included in the network construction to avoid making spurious associations. A motif enrichment analysis on the $2 \mathrm{~kb}$ upstream region of each gene was performed to predict possible regulatory interactions between the gene and TFs that bind to enriched cis-regulatory elements in its promoter region [32]. Network membership varied between male and female papaya flowers at different stages of development and generally increased over-development (Additional files 8, 9 and 10). Network size also varied between sexes at different stages, in which the male papaya flower network contained more co-expressed and co-regulated genes than the female flower network during the primordial (FS1) and mitotic (FS4) stages (Additional files 8 and 10). However, there were more co-expressed and coregulated genes in the female network during the meiosis (FS2-3) stage (Additional file 9). The average clustering coefficient, average path length, average degree, average connectivity, and total nodes of the papaya network for each period are shown in Additional file 11. The number of nodes and edges of the mitotic stage (F/MS4) network was much greater than that of the primordium stage $(\mathrm{F} /$ MS1) and the meiosis stage (F/MS2-3) network. In addition, the FS4 stage has the highest average degree and connectivity (Additional file 12), suggesting the existence of a more complex GRN than others.

$\mathrm{GO}$ term enrichment analysis of the genes in each network revealed key biological processes taking place during each stage of pistil development (Additional file 13). In female flowers, the genes associated with the primordium (FS1) stage were enriched for GO terms such as 'response to high light intensity', 'response to heat', and 'response to abiotic stimulus'. GO term enrichment analysis of genes expressed in female flowers during the meiosis (FS2-3) and mitotic (FS4) stages identified enrichment of many flower development-related GO terms such as 'carpel morphogenesis', 'floral organ morphogenesis,' 'meristem development/maintenance', 'organ morphogenesis', and 'plant organ development'. In male flowers, GO terms such as 'extracellular region,' 'cell wall', and 'tryptophan metabolic process' became enriched during the primordium (MS1) stage. Genes associated with meiosis (MS2-3) and mitotic (MS4) stages showed enrichment of GO terms such as 'response to biotic stimulus', 'external encapsulating structure', 'cation transmembrane transport', and 'ion transport'.

\section{Construction of a coregulatory subnetwork for pistil abortion}

To further explore the functions of important genes in the network, nodes with degree values in the top $10 \%$ were selected as candidate hub genes for further analysis. When the degree value of a gene is higher, it indicates that it is higher connectivity in the network, potentially indicating its importance in the regulatory network structure. A total of 1297 candidate hub genes were identified 
in six networks, including 70 in FS1, 122 in FS2-3, 587 in FS4, 11 in MS1, 248 in MS2-3, and 259 in MS4, of which 1056 genes are unique and 117 genes share at least two networks. Then, genes specifically expressed in female or male flowers at three different stages were identified $(0<$ FPKM $<1=$ no expression, FPKM $>1=$ expression $)$. 18 TFs as female-specific expressed genes and 7 TFs as male-specific expressed genes were identified that could play important roles in sex-specific regulation of pistil development (Table 1 and Additional file 15). We also investigated the expression of these $25 \mathrm{TFs}$ in $1-6 \mathrm{~mm}$ mixed flowers of different sex types [33]. Among them, the expression patterns of 18 TFs are consistent, and the remaining 7 TFs are expressed in mixed flowers of females and males. This indicates that these TFs specifically expressed in the primordium stage may also are involved in the growth and development of other floral organs except besides carpels (Additional file 15). A subnetwork was constructed using the network neighborhood around these key TF nodes. The CPHEC2, CpSUPL, and $C p A G L 11$ genes were expressed specifically during all three stages in females and were assigned a central role in the subnetwork (Fig. 4). Experimental verification of the expression of these three genes in female flowers by RT-qPCR was consistent with the results of our RNASeq data analysis (Additional file 14). The combined functions of individual TFs, any interconnected TFs, and protein-coding genes should be taken into account when describing the regulation of flower development.

KEGG pathway analysis of the CPHEC2, CPSUPL, and $C p A G L 11$ subnetwork genes revealed that expressed genes were enriched for pathways related to plant hormone signal transduction (Fig. 5a). Exploration of plant signal transduction pathways revealed differentially expressed genes associated with tryptophan metabolism (auxin) pathways and brassinosteroid signal transduction pathways (Fig. 5b). In the tryptophan metabolism pathway, $C p L A X 2, C P I A A 8$, and $C p M P$ showed higher expression in females than in males and their expression gradually increased during each of the three successive stages of pistil development. In the brassinosteroid biosynthesis pathway, the overall expression of $C p B S K 3$, $\mathrm{CpTCH} 4$ and $\mathrm{CpCYCD} 3$ was also higher in females than in males (Fig. 5b and Additional file 15). Further, we analyzed differences in the expression of genes related to auxin and brassinosteroid biosynthesis using Tukey's

Table 1 Information of stage-specific expression hub genes in the female and male gene regulatory network

\begin{tabular}{|c|c|c|c|c|c|c|}
\hline Gene ID & Female & Male & Symbol & Arabidosis homolog & Stage & Function \\
\hline sunup.05G0006810 & EXP & NO & CpAGL6 & AT2G45650 & FS2-3 & Flowing time \\
\hline sunup.04G0000360 & EXP & NO & CpHEC2 & AT3G50330 & FS1/FS2-3/FS4 & Gynoecium development \\
\hline sunup.03G0006060 & EXP & NO & CpSUPL & AT3G23130 & FS1/FS2-3/FS4 & Carpel development \\
\hline sunup.01G0026370 & EXP & NO & CpAGL11 & AT4G09960 & FS1/FS2-3/FS4 & Carpel and ovule development \\
\hline sunup.01G0022430 & EXP & NO & CpSVPL & AT4G24540 & FS1 & Flowing \\
\hline sunup.02G0012880 & EXP & NO & CpTGA9 & AT1G08320 & FS2-3/FS4 & Flower development \\
\hline sunup.01G0000330 & EXP & NO & CpCYC & AT1G67260 & FS2-3/FS4 & $\begin{array}{l}\text { Longitudinal elongation of petioles, rosette leaves } \\
\text { and inflorescent stems }\end{array}$ \\
\hline sunup.04G0001590 & EXP & NO & CpDOF5.7L & AT5G65590 & FS4 & Guard cell differentiation \\
\hline sunup.04G0003750 & EXP & NO & CpHBI1L & AT2G18300 & FS4 & Cell elongation and proliferation \\
\hline sunup.03G0023770 & EXP & NO & CpZAT11 & AT2G42410 & FS4 & Flower growth and development \\
\hline sunup.05G0010320 & EXP & NO & CpMYC2 & AT4G00870 & FS4 & Jasmonate-Mediated Growth \\
\hline sunup.08G0004940 & EXP & NO & CPATH51 & AT5G03790 & FS2-3/FS4 & Organ proportions \\
\hline sunup.02G0001210 & EXP & NO & CpbHLH30 & AT1G68810 & FS4 & Leaf development \\
\hline sunup.02G0008550 & EXP & NO & CpWIP6 & AT1G13290 & FS4 & Shoot and root development \\
\hline sunup.02G0002680 & EXP & NO & CPPRE5L & AT3G28857 & FS2-3/FS4 & Cell Elongation and Plant Development \\
\hline sunup.09G0005320 & EXP & NO & CpWOX1 & AT3G18010 & FS2-3/FS4 & Leaf blade outgrowth and floral organ development \\
\hline sunup.03G0015290 & EXP & NO & CpSCL32 & AT3G49950 & FS4 & Regulate growth and flowering \\
\hline sunup.04G0013610 & EXP & NO & CpBLH4 & AT4G36870 & FS2-3/FS4 & Ovule morphogenesis \\
\hline sunup.03G0020730 & NO & EXP & CPMUTE & AT3G06120 & MS2-3 & Stomatal development \\
\hline sunup.06G0008830 & NO & EXP & CpAMS & AT2G16910 & MS2-3 & Tapetal cell development \\
\hline sunup.01G0006170 & NO & EXP & CpBZIP43L & AT5G38800 & MS1/MS4 & NA \\
\hline sunup.08G0003710 & NO & EXP & CpNAC90L & AT3G44350 & MS4 & NA \\
\hline sunup.02G0022760 & NO & EXP & CpDIV & AT5G58900 & MS1/MS4 & Vascular Development \\
\hline sunup.06G0024960 & NO & EXP & CpMYB5 & AT3G13540 & MS1/MS4 & Seed coat and endosperm layers formation \\
\hline
\end{tabular}




\section{S1 \\ S2-3 \\ S4 \\ Genes \\ TFs}

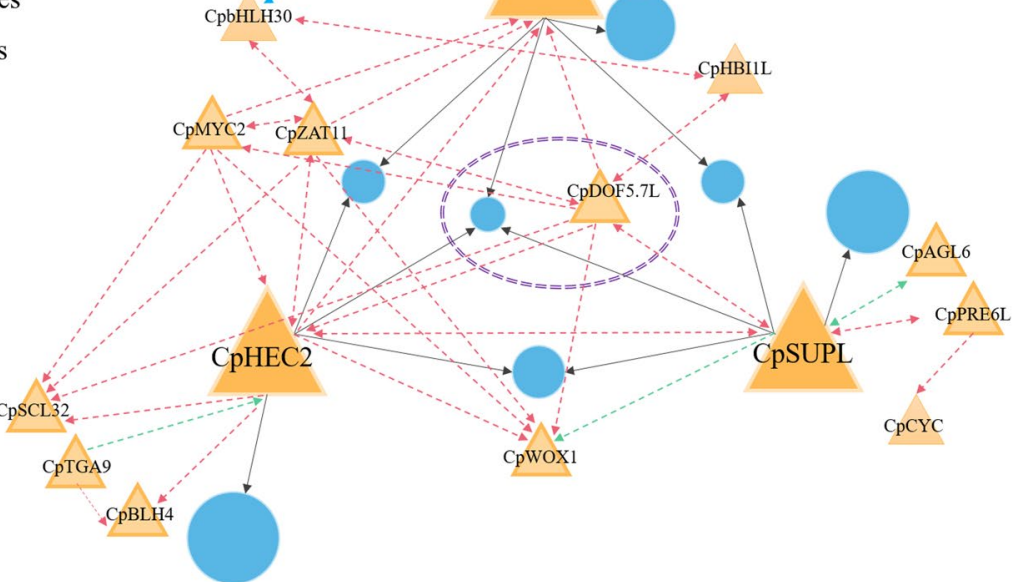

Fig. 4 The CPAGL11-CPSUPL-CPHEC2 regulatory subnetwork. The size of each blue circle represents the number of genes. The size of each triangle represents the importance of a transcription factor in the network, i.e., the degree of the node. The regulation between transcription factors is represented by dotted lines, and the solid black lines show the regulation of transcription factors on other genes. The arrow points represent the regulatory relationship between genes, and different colors are used at different developmental stages

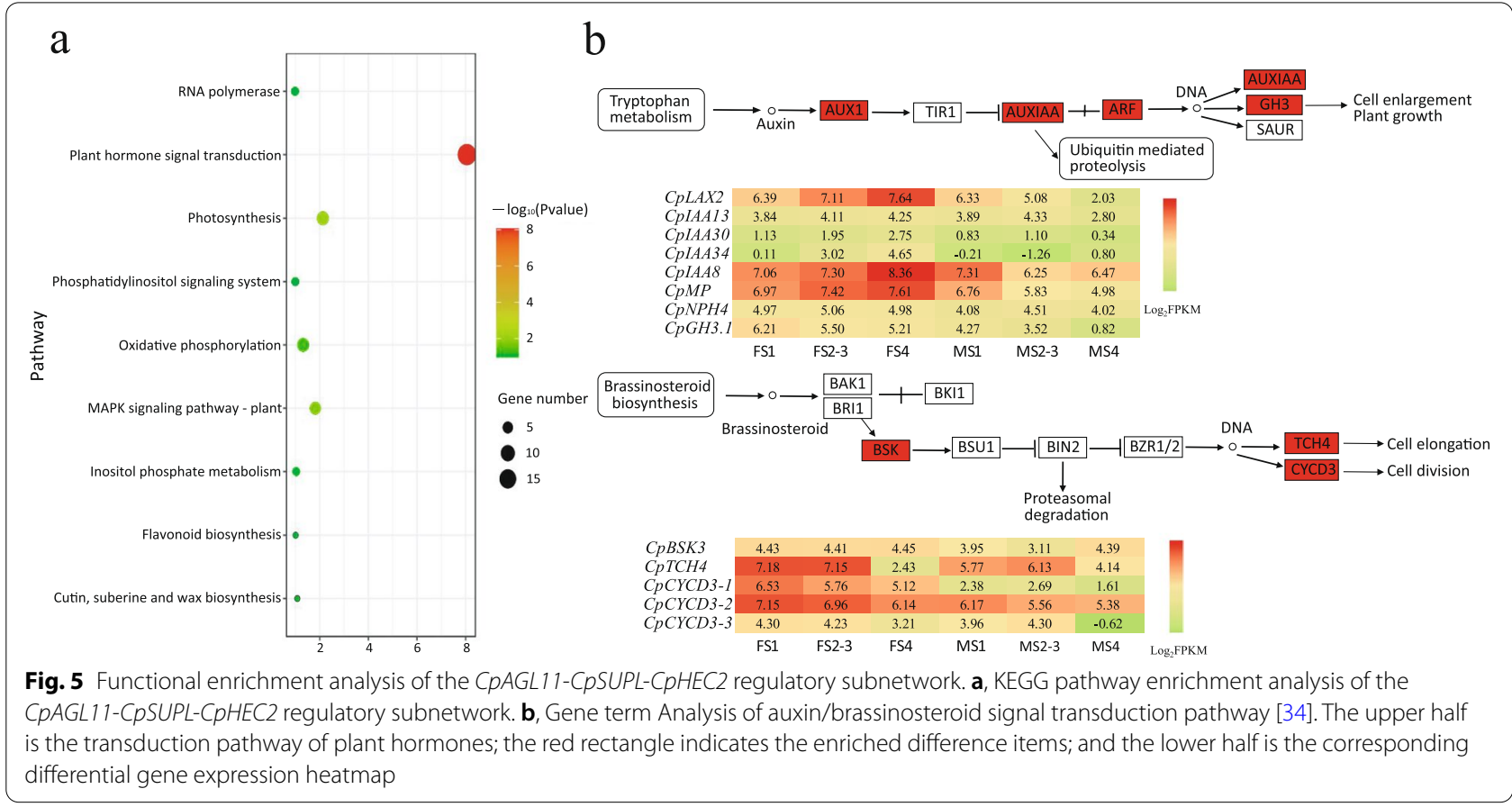

HSD and found significant differences in the expression of $C p C Y B 85 A 1, C p Y U C C A 4, C p T A R 2$ and CpNIT4 between male and female flowers at different stages of development (Fig. 6 and Additional file 15). CpCYB85A1 catalyzes the last reaction to produce brassinolide and converts 6-deoxycasterone into a seven-membered lactone ring [35]. CpNIT4 encodes a gene essential for the transformation of indole-3-acetonitrile (IAN) into 


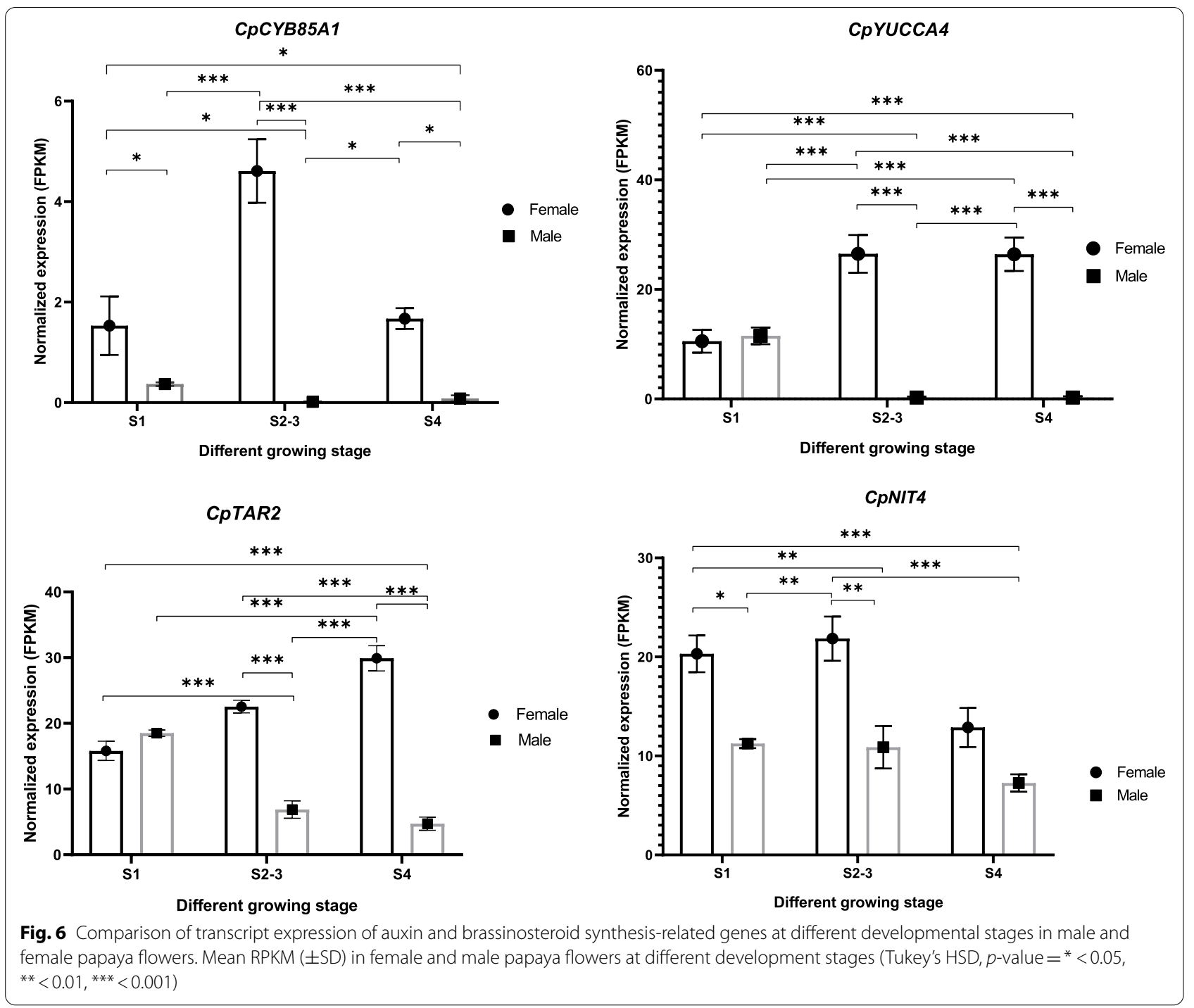

indole-3-acetamide (IAM) and indole-3-acetic acid (IAA) [36]. CpYUCC4 encodes a flavin monooxygenase, which is a possible bridge between auxin biosynthesis and pistil development $[37,38]$. In summary, we suggest that papaya pistil abortion might be caused by the lack of expression of some important TFs such as $C p H E C 2$, CPSUPL and CPAGL11 that are mainly involved in the auxin and brassinosteroid synthesis pathways.

\section{Discussion}

The construction of GRNs is useful for depicting gene interactions, and can also be used to predict possible regulatory relationships and modules within the network [39]. By performing transcriptomic analysis of male and female flowers from three stages in papaya, we constructed a series of networks underlying flower development in papaya. GO enrichment analysis of network members revealed that genes in the primordium stage were enriched mainly in some response-related pathways, such as light, heat and abiotic stimulus (Additional file 13). However, we did not identify any other genes or pathways in this stage that seemed to be related to sex determination. The results suggested that sex determination in female papaya flowers might already be established before the primordium stage as there was no trace of stamens, whereas sex determination in male flowers occurred at a later stage when the pistil development was aborted. At the S2-3 and S4 stages in females, many significantly enriched GO terms such as 'carpel morphogenesis', 'floral organ morphogenesis/development', 'plant organ development', and 'organ morphogenesis' were related to floral organ formation and development (Additional file 13). Importantly, genes involved in these pathways exhibited decreased expression or no expression in 
males (Table 1), suggesting that processes leading to pistil abortion in male flowers might begin at the meiosis stage or earlier.

TFs are essential regulators for the transcriptional control of gene expression, which is crucial for the development and growth of plants. Previous studies in various species discovered many TFs that are involved in plant growth, organ development, and response to biotic and abiotic stresses [40-42]. The identified several candidate TFs that might be crucial for the development of papaya flowers (Fig. 4 and Table 1). For instance, CpHEC2 is a homolog of the Arabidopsis thaliana gene HEC2 (AT3G50330), which belongs to the basic helix-loophelix (bHLH) TF family. In Arabidopsis, HEC and SPT act synergistically to regulate the development of the female reproductive tract and are probably involved in auxinmediated control of gynoecium patterning. Loss of $H E C$ gene function can result in defective transmitting tract, stigma, and septum, as well as reduced fertility [43]. Further, CPSUPL and CpAGL11 have already been shown to play an important role in carpel development in flowers $[8,11,13]$. Additionally, the other differentially expressed TFs in female papaya flowers were mainly related to the regulation of flowering. Taken together, these femalespecific TFs might play a pivotal role in regulating the differential floral development patterns of male and female papaya flowers.

Plant hormones act as signaling compounds that promote and regulate plant growth and development. Phytohormones influence many processes of floral and post-floral morphogenesis in angiosperms [44]. Various plant hormone signaling pathways control flower development by transmitting both endogenous and environmental signals to genes that directly control flowering [45]. The significant differences between males and females in the expression of genes related to plant hormone signal transduction suggested that the inhibition of plant hormone signal transduction might be an important cause of pistil abortion in male flowers (Fig. 5). In addition, some genes related to auxin and brassinosteroid synthesis are not expressed or are expressed at lower levels in male flowers were detected, in which pistils abort (Fig. 6). These findings provide a basis for further functional studies of plant hormone function in pistil development, which will improve our understanding of the molecular mechanisms driving the regulation of pistil development in papaya.

\section{Conclusions}

Discovering mechanisms for pistil formation and development has implications in dioecious crop improvement. The normal development of the pistil is essential to the reproduction of flowering plants. Papaya is a dioecious plant, but there is an aborted pistil in male flowers that can be restored sporadically under optimal environmental conditions. In this study, we divided the pistil into four developmental stages. The first stage (F/MS1) is the primordia period, the second and third stages (F/MS2 and F/MS3) are the meiotic period, and the fourth is the mitotic period. The transcriptome analysis of these stages revealed differentially expressed regulatory genes in transcription levels. A transcription factor regulatory network CpAGL11-CpSUPL-CpHEC2 was constructed. The specific expression of some transcription factors closely related to pistil development in female flowers also were detected. Further functional analysis showed that the differential gene expression in phytohormones signaling pathway (mainly auxin and brassinosteroid) and auxin/ brassinosteroid biosynthesis could be the main reason leading to pistil abortion.

\section{Methods \\ Morphological observation and sampling of the pistil development stage}

Female and male flowers of papaya were sampled and fixed according to flower size and length and stored in FAA liquid. After putting samples through an alcohol dehydration series, flower samples were embedded in paraffin LEICA EG $1150 \mathrm{H}$ ) in a Leica EG $1150 \mathrm{H}$ paraffin embedding apparatus (Leica, Germany) at a melting point of $50-52^{\circ} \mathrm{C}$. Transverse sections with a thickness of $10 \mu \mathrm{m}$ were made on a Leica automatic rotary microtome and stained with Brilliant Green (BIO-RAD). Sections were observed and imaged under an Olympus Model BH2 optical microscope (Olympus, Japan). For the specific methods used for paraffin embedding and sectioning, please refer to [46].

The sectioned and embedded papaya flowers were dissected under a dissecting microscope (Olympus, Japan) to isolate the different developmental stages of the male and female papaya flowers. Due to the difficulty in sampling pistils from male and female flowers at the primordium stage, the flower buds at the primordium stage was used as the experimental material for this study. Each sample comprised three biological replicates, each consisting of multiple pistils.

\section{RNA extraction, library construction and sequencing}

The different tissue samples were placed in TRIzol ${ }^{\circledR}$ Reagent (Invitrogen), and total RNAs were extracted according to the TRIzol manufacturer's instructions. Residual genomic DNA (gDNA) in the RNA samples was then removed by DNase treatment (DNase I RNase-free, Thermo Scientific, USA). An Agilent 2100 BioAnalyzer (Agilent Technologies) was used to determine the quality and integrity of RNA. High-quality RNA samples with 
no signs of degradation are used to prepare RNA-Seq libraries.

Each RNA-Seq library was constructed separately using the NEBNext Ultra RNA Library Prep Kit for Illumina (New England Biolabs) according to the manufacturer's protocol. The library was evaluated by electrophoresis ( $0.8 \%$ agarose, TBE $1 \mathrm{X}$ buffer), and quantified with a fluorometer (Qubit reagent fluorometer, Invitrogen, USA). A total of $18 \mathrm{cDNA}$ libraries were sequenced on an Illumina platform (HiSeq2500) to generate a larger number of paired-end sequence reads (150 nt). Raw RNA sequence data for each library is publicly available on NCBI BioProject, under the accession number: PRJNA687615 (https:// www.ncbi.nlm.nih.gov/bioproject/PRJNA687615). Besides these libraries, RNA sequencing data from normal female buds (Accession number: PRJNA532376), normal female leaves (Accession number: SRX6473537 and SRX6473538), normal male buds (Accession number: PRJNA532376), and normal male leaves (Accession number: SRX6473540 and SRX6473541), were downloaded from the Sequence Read Archive (SRA) database [47]. The information of the analyzed library is presented in Additional file 16.

\section{Read mapping and gene expression analysis}

The raw data were filtered by removing adapters and lowquality bases using Trimmomatic [48]. The clean highquality reads were then mapped to the papaya reference genome [49] using the Bowtie2 [50] with the end-to-end model default parameter setting. FPKM (Fragments Per Kilobase pair per Million reads) values were calculated using RSEM [51]. The SCC algorithm was used to calculate the correlations between experimental values obtained from two or three biological replicates of samples. PCA analysis was performed using the $\mathrm{R}$ function prcomp and plotted using the pca3d package [52], respectively.

In this study, Z-score was used to represent relative gene expression [53]. The Z-score of the gene $i$ in the stage or sample $j$ is determined by the equation $z_{i j}=\left(x_{i j}\right.$ $\left.\mu_{\mathrm{i}}\right) / S D_{i}$, where $\mathrm{x}$ is the expression value FPKM, and $\mu$ and $S D$ are the mean and standard deviation of the sample, respectively.

\section{Identification of preferentially expressed genes}

The preferentially expressed genes in different stages or samples were defined using the SS scoring algorithm [54, 55], which compares the expression of a gene in a specific stage or sample with its maximum expression level in all other stages or samples. The expression value of a gene $i$ in 10 samples is denoted as $E V_{i}=\left(E_{1}^{i}, E_{2,}^{i}, E_{3,}^{i}, E_{4 \ldots,}^{i} E_{9}^{i}\right.$, $\left.E^{i}{ }_{10}\right)$, so the SS score of gene $i$ in sample or stage $j$ was calculated as: $S S(i, j)=1-\left(\max E_{s}^{i} / E_{j}^{i}\right)$, where $1 \leq s \leq 10, s \neq j$.
The higher the SS score of a gene at a particular stage, the higher the possibility of preferential expression of the gene at this stage. In this study, a gene with an SS score greater than 0.3 was defined as preferentially expressed in that sample or stage.

\section{Analysis of differential gene expression}

The DESeq2 [30] and edgeR [31] with default parameters in $\mathrm{R}$ were used to calculate differential expression of genes. The differential expressed genes (DEGs) were designated as those with FDR adjusted $p<0.05$ and $\mid \log 2$ fold change $\mid>1$.

\section{GO term and KEGG pathway enrichment analysis}

Subsequently, analysis of the potential functions of differentially expressed genes was performed in two ways. Gene ontology enrichment analysis was performed using TBtools [56]. The $p$-value was calculated for each enriched GO term, and terms with $p$-value $\leq 0.05$ were considered meaningfully enriched. KEGG pathway enrichment analysis was performed using the R2HTML package in R. Similarly, $p$-value $\leq 0.05$ was also the criterion for meaningful pathway enrichment. The bubble charts for GO term and KEGG pathway enrichment were drawn using ggplot 2 in the $\mathrm{R}$ package.

\section{Gene co-expression construction}

Mutual rank (MR) analysis [57] was used to identify significant co-expression relationships between DEGs in papaya pistils. Pearson Correlation Coefficients (PCC) were determined for all DEGs from papaya pistils in this study using $\mathrm{R}$. The stringent criterion for designating positively or negatively correlated gene expression was set at $\geq|0.95|$. The MR was calculated from the PCC rank, and geometric means were taken between the PCC rank of gene $A$ to gene $B$ and that of gene $B$ to gene $A$. $\mathrm{MR}$ value ranged from 0 to 1 , with 1 representing the most significant interaction. All MR values of $>=0.95$ for interactions were included in construction of a coexpression network.

PlantPAN [58] was used to identify genes encoding transcription factors in the whole papaya genome and detected a total of 1475 genes encoding putative transcription factors for downstream analysis. In this study, the promoter sequences were defined as the $2 \mathrm{~Kb}$ upstream of the predicted Transcription Start Site (TSS) for every gene in the papaya genome were retrieved from papaya reference genome [49]. The database of known transcription factor binding sites at JASPAR Plantae [59] (http://jaspar.genereg.net/) and the PlantTFDB motif database [60] (http://planttfdb.gao-lab.org/) were used to predict the cis-regulatory elements (CREs) in target gene promoter regions. Promoter sequences of DEGs were analyzed for statistical overrepresentation of CREs 
for particular TF families using a MEME suite tool called AME [61]. The significance of motif enrichment was tested using Fisher's exact test with a Bonferroni correction for multiple comparisons. A $p$-value $<0.001$ for a candidate motif was considered a meaningful enrichment, so that CRE would then be considered included in construction of the gene regulatory network. The final networks contained highly significant interactions $(\mathrm{MR}>=0.95)$ between pairs of differentially expressed genes with predicted binding interactions between TF and target genes (DEGs). The resulting network was visualized using Cytoscape version 3.6.1 [62].

\section{RT-qPCR analysis}

We used RT-qPCR to validata false positives of RNASeq analysis. The primers were designed based on gene model sequences (Additional file 17). The $1 \mu \mathrm{g}$ RNA as a template for reverse transcription using PrimeScript First Strand cDNA Synthesis Kit (TaKaRa). Then the synthesized first-strand cDNA was diluted 10 -fold, and $1 \mu \mathrm{l}$ of the diluted cDNA was used for real-time quantitative PCR (qPCR reaction). The papaya TATA binding protein 2 (TBP2) genes were included as internal reference genes for normalization [63]. Each RT-qPCR analysis was performed in triplicate. The $2^{-\triangle \triangle \mathrm{Ct}}$ method was used to calculate the relative expression [64].

\section{Method declaration}

All analysis methods are implemented in accordance with relevant guidelines and regulations in this manuscript.

\section{Supplementary Information}

The online version contains supplementary material available at https://doi. org/10.1186/s12864-021-08197-7.

Additional file 1. The heatmap of the biological repeat correlation analysis of RNA-seq data samples. The suffixes 1,2, and 3 represent three repeats, and the leaf sample has only two repeats. F, Female; M, Male; S, stage; L, leaf; FS1, Female stage1; and so on.

Additional file 2. Global transcript analysis in different stages/samples. a, Count the expression ratio of high/medium/low expressed genes. Percentage is used to indicate the number of genes at different expression levels $b$, Comparison of the number of expressed genes between females and males. The height of the histogram represents the number of gene expressions in different stages.

Additional file 3. Statistics of genes preferentially expressed by different sexes at different developmental stages.

Additional file 4. Statistics of transcription factors preferentially expressed in different stages.

Additional file 5. Comparison of gene expression in RT-qPCR experiment and RNA-seq data analysis. a, Female samples. b, Male samples.

Additional file 6. Count the differentially expressed genes of different methods.
Additional file 7. Bar chart of differentially expressed transcription factors between females and males at different stages. The abscissa shows the different transcription factor families. Different colors represent different stages of pistil development.

Additional file 8. Diagram of gene regulation network in primordium stage (S1), red triangles represent transcription factors, blue circles represent other genes. The black lines link the regulatory relationships between genes a, Female sample. b, Male sample.

Additional file 9. Diagram of gene regulation network in meiosis stage (S2-3), red triangles represent transcription factors, blue circles represent other genes. The black line represents the regulatory relationship. a, Female sample. b, Male sample.

Additional file 10. Diagram of gene regulation network in mitosis stage (S4), red triangles represent transcription factors, blue circles represent genes. Transcription factors and regulated genes are connected with black lines. a, Female sample. b, Male sample.

Additional file 11. Statistics of topological attribute information of different gene regulatory networks.

Additional file 12. Statistics information of each node in the network from six gene regulation networks.

Additional file 13. Bubble chart of $\mathrm{GO}$ enrichment analysis of gene regulatory network in different stages. Different bubbles visually show the sharing and unique pathways of different networks.

Additional file 14. RT-qPCR to verify the expression pattern of CpHEC2, CPSUPL, and CPAGL11 genes. The Y coordinate represents the normalized expression, and the $\mathrm{X}$ coordinate represents different developmental stages.

Additional file 15. The expression information of important genes at different developmental stages in this study.

Additional file 16 . Summary statistics of RNA-Seq data from papaya male and female flowers.

Additional file 17. Primer information used for RT-qPCR experimental verification in this study. Primers CpHEC2, CpSUPL, and CPAGL11 are used to verify the false positive of RNA-seq differential expression analysis. FS and MS primers are used to verify the false positives of the female and male preferential expression algorithm, respectively.

\section{Acknowledgements}

This work was supported by startup fund from Fujian Agriculture and Forestry University.

\section{Authors' contributions}

R.M. designed the project. Z.L. performed data analysis and experiments. F.D. drew the gene regulatory network (Fig. 4 and Additional files 8, 9 and 10) and wrote the results, J.L. performed paraffin section experiment (Fig. 1). L.X performed RT-qPCR verification. Z.L., A.M.-C., and R.M. wrote and revised the manuscript. All authors reviewed the manuscript. The author(s) read and approved the final manuscript.

\section{Funding}

This project was supported by US National Science Foundation (NSF) Plant Genome Research Program Award DBI-1546890.

\section{Availability of data and materials}

The pistil RNA-seq raw data was uploaded to NCBI BioProject, under the accession number: PRJNA687615 (https://www.ncbi.nlm.nih.gov/bioproject/PRJNA 687615).

\section{Declarations}

Ethics approval and consent to participate Not applicable. 


\section{Consent for publication \\ Not applicable.}

\section{Competing interests}

The authors declare that they have no conflict of interest.

\section{Author details}

${ }^{1}$ Center for Genomics and Biotechnology, Fujian Provincial Key Laboratory of Haixia Applied Plant Systems Biology, Fujian Agriculture and Forestry University, Fujian 350002 Fuzhou, China. ${ }^{2}$ The Wuhan Botanical Garden of the Chinese Academy of Sciences, Wuhan 430074, Hubei, China. ${ }^{3}$ Department of Plant Biology, University of Illinois at Urbana-Champaign, Urbana, IL 61801, USA

\section{Received: 6 January 2021 Accepted: 15 November 2021}

Published online: 05 January 2022

\section{References}

1. Tao J, Liang W, An G, Zhang D. OsMADS6 controls flower development by activating rice FACTOR OF DNA METHYLATION LIKE1. Plant Physiol. 2018;177(2):713-27.

2. Prunet N, JackTP. Flower development in Arabidopsis: there is more to it than learning your ABCs. Flower Dev. 2014:3-33.

3. Coen ES, Meyerowitz EM. The war of the whorls: genetic interactions controlling flower development. Nature. 1991;353(6339):31-7.

4. Rijpkema AS, Vandenbussche M, Koes R, Heijmans K, Gerats T. Variations on a theme: changes in the floral ABCs in angiosperms. Semin Cell Dev Biol. 2010:100-7.

5. Yanofsky MF, Ma H, Bowman JL, Drews GN, Feldmann KA, Meyerowitz EM. The protein encoded by the Arabidopsis homeotic gene agamous resembles transcription factors. Nature. 1990;346(6279):35-9.

6. Smaczniak C, Immink RG, Angenent GC, Kaufmann K. Developmental and evolutionary diversity of plant MADS-domain factors: insights from recent studies. Development. 2012;139(17):3081-98.

7. Pinyopich A, Ditta GS, Savidge B, Liljegren SJ, Baumann E, Wisman E, et al. Assessing the redundancy of MADS-box genes during carpel and ovule development. Nature. 2003;424(6944):85-8.

8. Lu H, Klocko AL, Brunner AM, Ma C, Magnuson AC, Howe GT, et al. RNA interference suppression of AGAMOUS and SEEDSTICK alters floral organ identity and impairs floral organ determinacy, ovule differentiation, and seed-hair development in Populus. New Phytol. 2019;222(2):923-37.

9. Busi MV, Bustamante C, D’angelo C, Hidalgo-Cuevas M, Boggio SB, Valle EM, et al. MADS-box genes expressed during tomato seed and fruit development. Plant Mol Biol. 2003:52(4):801-15.

10. Ocarez N, Mejía N. Suppression of the D-class MADS-box AGL11 gene triggers seedlessness in fleshy fruits. Plant Cell Rep. 2016;35(1):239-54

11. Xu Y, Prunet N, Gan ES, Wang Y, Stewart D, Wellmer F, et al. SUPERMAN regulates floral whorl boundaries through control of auxin biosynthesis. EMBO J. 2018;37(11):e97499.

12. Skinner DJ, Hill TA, Gasser CS. Regulation of ovule development. Plant Cell. 2004;16(suppl 1):S32-45.

13. Kazama Y, Fujiwara MT, Koizumi A, Nishihara K, Nishiyama R, Kifune E, et al. A SUPERMAN-like gene is exclusively expressed in female flowers of the dioecious plant Silene latifolia. Plant Cell Physiol. 2009;50(6):1127-41.

14. Xu K, Wang L, Liu N, Xie X, Zhu Y. Characterization of a SUPERMAN-like gene, MdSUP11, in apple (Malus $\times$ domestica Borkh.). Plant Physiol Biochem. 2018;125:136-42.

15. Yan Y, Christensen S, Isakeit T, Engelberth J, Meeley R, Hayward A, et al. Disruption of OPR7 and OPR8 reveals the versatile functions of jasmonic acid in maize development and defense. Plant Cell. 2012:24(4):1420-36.

16. Acosta IF, Laparra H, Romero SP, Schmelz E, Hamberg M, Mottinger JP, et al. Dellaporta SL: tasselseed1 is a lipoxygenase affecting jasmonic acid signaling in sex determination of maize. Science. 2009;323(5911):262-5.

17. Boualem A, Troadec C, Camps C, Lemhemdi A, Morin H, Sari MA, et al. A cucurbit androecy gene reveals how unisexual flowers develop and dioecy emerges. Science. 2015;350(6261):688-91.
18. Huang HY, Jiang WB, Hu YW, Wu P, Zhu JY, Liang WQ, et al. BR signal influences Arabidopsis ovule and seed number through regulating related genes expression by BZR1. Mol Plant. 2013;6(2):456-69.

19. Fujioka S, Li J, Choi YH, Seto H, Takatsuto S, Noguchi T, et al. The Arabidopsis deetiolated 2 mutant is blocked early in brassinosteroid biosynthesis. Plant Cell. 1997;9(11):1951-62.

20. Barro-Trastoy D, Carrera E, Baños J, Palau-RodríguezJ R-RO, Tornero $P$, Alonso JM, et al. Regulation of ovule initiation by gibberellins and brassinosteroids in tomato and Arabidopsis: two plant species, two molecular mechanisms. Plant J. 2020;102(5):1026-41.

21. Liu X, Li Y, Zhao Y, Zhang X. Effects of auxin on the carpel development of Capsella bursa-pastoris. J Henan Agric Sci. 2018;47(8):88-94.

22. Zhu Z. The PIN genes expression and its relationship to carpel morphogenesis of Capsella bursa-pastoris: Hunan Agricultural University; 2013.

23. Qing Y. The analyses of PIN3 of Capsella bursa-pastoris and its influence to carpel morphogenesis: Hunan Agricultural University; 2014.

24. Dufaÿ M, Champelovier P, Käfer J, Henry JP, Mousset S, Marais G. An angiosperm-wide analysis of the gynodioecy-dioecy pathway. Ann Bot. 2014;114(3):539-48.

25. Liu Z, Moore PH, Ma H, Ackerman CM, Ragiba M, Yu Q, et al. A primitive $Y$ chromosome in papaya marks incipient sex chromosome evolution. Nature. 2004;427(6972):348-52.

26. Wang J, Na JK, Yu Q, Gschwend AR, Han J, Zeng F, et al. Sequencing papaya $\mathrm{X}$ and $\mathrm{Yh}$ chromosomes reveals molecular basis of incipient sex chromosome evolution. Proc Natl Acad Sci. 2012;109(34):13710-5.

27. Gschwend AR, Yu Q, Tong EJ, Zeng F, Han J, VanBuren R, et al. Rapid divergence and expansion of the X chromosome in papaya. Proc Natl Acad Sci. 2012;109(34):13716-21.

28. Liao Z, Yu Q, Ming R. Development of male-specific markers and identification of sex reversal mutants in papaya. Euphytica. 2017;213(2):53.

29. Ming R, Bendahmane A, Renner SS. Sex chromosomes in land plants. Annu Rev Plant Biol. 2011;62:485-514.

30. Love Ml, Huber W, Anders S. Moderated estimation of fold change and dispersion for RNA-seq data with DESeq2. Genome Biol. 2014;15(12):550.

31. Robinson MD, McCarthy DJ, Smyth GK. edgeR: a bioconductor package for differential expression analysis of digital gene expression data. Bioinformatics. 2010;26(1):139-40.

32. Hetti-Arachchilage M, Mullet JE, Marshall-Colón A. Sorghum bicolor cultivars have divergent and dynamic gene regulatory networks that control the temporal expression of genes in stem tissue. bioRxiv. 2020:1-47.

33. Zerpa-Catanho D, Wai J, Wang ML, Nguyen J, Ming R. Differential gene expression among three sex types reveals a MALE STERILITY1 (CPMS1) for sex differentiation in papaya. BMC Plant Biol. 2019;19:545.

34. Kanehisa M, Goto S, Sato Y, Furumichi M, Tanabe M. KEGG for integration and interpretation of large-scale molecular data sets. Nucleic Acids Res. 2012:40(D1):D109-14.

35. Kim TW, Chang SC, Lee JS, Takatsuto S, Yokota T, Kim SK. Novel biosynthetic pathway of castasterone from cholesterol in tomato. Plant Physiol. 2004;135(3):1231-42.

36. Piotrowski M. Primary or secondary? Versatile nitrilases in plant metabolism. Phytochemistry. 2008:69(15):2655-67.

37. Robert HS, Crhak Khaitova L, Mroue S, Benková E. The importance of localized auxin production for morphogenesis of reproductive organs and embryos in Arabidopsis. J Exp Bot. 2015;66(16):5029-42.

38. Munguía-Rodríguez AG, López-Bucio JS, Ruiz-Herrera LF, Ortiz-Castro R, Guevara-García ÁA, Marsch-Martínez N, et al. YUCCA4 overexpression modulates auxin biosynthesis and transport and influences plant growth and development via crosstalk with abscisic acid in Arabidopsis thaliana. Genet Mol Biol. 2020;43(1):e20190221.

39. Wang J, Yu H, Weng X, Xie W, Xu C, Li X, et al. An expression quantitative trait loci-guided co-expression analysis for constructing regulatory network using a rice recombinant inbred line population. J Exp Bot. 2014;65(4):1069-79.

40. Groszmann M, Bylstra Y, Lampugnani ER, Smyth DR. Regulation of tissuespecific expression of SPATULA, a bHLH gene involved in carpel development, seedling germination, and lateral organ growth in Arabidopsis. J Exp Bot. 2010;61(5):1495-508.

41. Wang L, Li Y, Jin X, Liu L, Dai X, Liu Y, et al. Floral transcriptomes reveal gene networks in pineapple floral growth and fruit development. Commun Biol. 2020;3(1):1-15. 
42. Liao Y, Zou HF, Wang HW, Zhang WK, Ma B, Zhang JS, et al. Soybean GmMYB76, GmMYB92, and GmMYB177 genes confer stress tolerance in transgenic Arabidopsis plants. Cell Res. 2008;18(10):1047-60.

43. Gremski K, Ditta G, Yanofsky MF. The HECATE genes regulate female reproductive tract development in Arabidopsis thaliana. Development. 2007;134(20):3593-601.

44. Khan MR, Hu J, He C. Plant hormones including ethylene are recruited in calyx inflation in Solanaceous plants. J Plant Physiol. 2012;169(10):940-8.

45. Conti L. Hormonal control of the floral transition: can one catch them all? Dev Biol. 2017;430(2):288-301.

46. Ao CQ. Developmental origins of the conjoined twin mature embryo sacs in Smilax davidiana, with special notes on the formation of their embryos and endosperms. Am J Bot. 2013;100(12):2509-15.

47. Kodama Y, Shumway M, Leinonen R. The sequence read archive: explosive growth of sequencing data. Nucleic Acids Res. 2012;40(D1):D54-6.

48. Bolger AM, Lohse M, Usadel BJ. Trimmomatic: a flexible trimmer for Illumina sequence data. Bioinformatics. 2014;30(15):2114-20.

49. Ming R, Hou S, Feng Y, Yu Q, Dionne-Laporte A, Saw JH, et al. The draft genome of the transgenic tropical fruit tree papaya (Carica papaya Linnaeus). Nature. 2008;452(7190):991-6.

50. Langmead B, Salzberg SL. Fast gapped-read alignment with bowtie 2. Nat Methods. 2012;9(4):357.

51. Li B, Dewey CN. RSEM: accurate transcript quantification from RNASeq data with or without a reference genome. BMC Bioformatics. 2011;12(1):323.

52. Holand SM. Principal components analysis (PCA). Department of Geology. Athens: University of Georgia; 2008.

53. Cheadle C, Vawter MP, Freed WJ, Becker KG. Analysis of microarray data using Z score transformation. J Mol Diagn. 2003;5(2):73-81.

54. Ma C, Xin M, Feldmann KA, Wang X. Machine learning-based differential network analysis: a study of stress-responsive transcriptomes in arabidopsis. Plant Cell. 2014;26(2):520-37.

55. Zhan J, Thakare D, Ma C, Lloyd A, Nixon NM, Arakaki AM, et al. RNA sequencing of laser-capture microdissected compartments of the maize kernel identifies regulatory modules associated with endosperm cell differentiation. Plant Cell. 2015;27(3):513-31.

56. Chen $\mathrm{C}$, Chen $\mathrm{H}$, Zhang Y, Thomas HR, Frank MH, He Y, et al. TBtools-an integrative toolkit developed for interactive analyses of big biological data. Mol Plant. 2020;13(8):1194-202.

57. Obayashi T, Kinoshita K. Rank of correlation coefficient as a comparable measure for biological significance of gene coexpression. DNA Res. 2009;16(5):249-60.

58. Chow CN, Zheng HQ, Wu NY, Chien CH, Huang HD, Lee TY, et al. PlantPAN 2.0: an update of plant promoter analysis navigator for reconstructing transcriptional regulatory networks in plants. Nucleic Acids Res. 2016;44(D1):D1154-60.

59. Sandelin A, Alkema W, Engström P, Wasserman WW, Lenhard B. JASPAR: an open-access database for eukaryotic transcription factor binding profiles. Nucleic Acids Res. 2004;32(suppl_1):D91-4.

60. Jin J, Tian F, Yang DC, Meng YQ, Kong L, Luo J, et al. PlantTFDB 4.0: toward a central hub for transcription factors and regulatory interactions in plants. Nucleic Acids Res. 2016:45:982.

61. Bailey TL, Johnson J, Grant CE, Noble WS. The MEME suite. Nucleic Acids Res. 2015;43(W1):W39-49.

62. Smoot ME, Ono K, Ruscheinski J, Wang PL, Ideker T. Cytoscape 2.8: new features for data integration and network visualization. Bioinformatics. 2011;27(3):431-2.

63. Zhu X, Li X, Chen W, Chen J, Lu W, Chen L, et al. Evaluation of new reference genes in papaya for accurate transcript normalization under different experimental conditions. PLoS One. 2012;7(8):e44405.

64. Livak KJ, Schmittgen T. Analysis of relative gene expression data using real-time quantitative $P C R$ and the $2-\triangle \triangle C T$ method. Methods 2001;25(4):402-8.

\section{Publisher's Note}

Springer Nature remains neutral with regard to jurisdictional claims in published maps and institutional affiliations.

Ready to submit your research? Choose BMC and benefit from:

- fast, convenient online submission

- thorough peer review by experienced researchers in your field

- rapid publication on acceptance

- support for research data, including large and complex data types

- gold Open Access which fosters wider collaboration and increased citations

- maximum visibility for your research: over $100 \mathrm{M}$ website views per year

At BMC, research is always in progress.

Learn more biomedcentral.com/submissions 